\title{
Exposing Strings in the Laboratory with a Novel Technique
}

\author{
AC Tahan \\ PO Box 391987, Cambridge, MA 02139, USA \\ E-mail: actahan@yahoo.com
}

Received: March 16, 2011

Accepted: April 8, $2011 \quad$ Published: November 1, 2011

doi:10.5539/apr.v3n2p39

URL: http://dx.doi.org/10.5539/apr.v3n2p39

\begin{abstract}
String theorists have proposed that multi-national collider experiments could provide evidence for strings. The scientific community generally has been more reserved, believing possible detections would be indirect at best. Based on the theoretical prediction that if in existence strings should have resonance frequencies, this paper shows a novel technique involving Hydrogen in a magnetic field that led to the first direct data for strings, particularly a picture of a multi-dimensional string D-brane. This paper introduces string experimentation, the ability to study strings directly in the laboratory.
\end{abstract}

Keywords: Strings, Brane, Quantum gravity (graviton), Black hole

\section{Introduction}

Scientists feel that evidence for strings can emerge only through high energy studies. The research for this paper used a low energy method. The multi-dimensional D-brane that appeared looked similar to what theorists have predicted (Thorlacius, L. 1998). Numerous papers on D-branes can be referenced; certain citations can be replaced by multiple, similar manuscripts. Videlicet, this work is not intended to suggest a new type of visualization but instead confirms string theory that previously has been only a mathematical concept. What must be understood is that the process to expose strings experimentally was gradual. Trials would start with low energy but a high energy could eventually be reached; thus, interpretation as a high or low energy work would depend on where in a study the description would be made.

This manuscript is an uncommon study of spacetime on Earth. Spacetime is mentioned in the correct way of being ubiquitous, not simply outside of the Earth atmosphere as typically imagined and studied. Since string theory is well-accepted by theorists to be a theory for quantum gravity (Scherk, J. \& Schwarz, J.H. Phys. Letters 1974), an observed curvature of spacetime in the laboratory that accompanied the D-brane was considered due to gravitons.

\section{Background}

Strings have been written should have tension and magnetic properties (Zwiebach, B. 2004), which suggests that the tension could be altered in a magnetic field. Research with various magnetic fields and a range of electromagnetic frequencies led to the hypothesis that by using specific field strengths, certain low frequencies at approximate amplitudes would be resonance frequencies for strings. Preliminary work involving passing laser light near a reaction vessel graphite tube (Crucible, Saed/Manfredi G40, 1.5"OD x 1.25"ID x 3.75"DP) where extremely low frequency radio waves interacted with sulfuric acid $\left(\mathrm{H}_{2} \mathrm{SO}_{4}\right)$, usually $20 \mathrm{~mL}$, in a static magnetic field (typically 2000Gauss (Gs), General Electric type 15A 270) resulted in video captures of the light being pushed and pulled in relation to the position of the vessel. Influences from the tube were thought causing the bending of nearby spacetime and consequently the light passing in the area.

To understand what might be bending spacetime that resulted in the unusual light observations, the hypothesis was expanded to consider that the laser light should curve around the holding vessel if gravitons had been produced due to the resonance frequencies and had been the reason for the bending. The idea considered gravitons to be vibrating string bodies, a carrier of a specific resonance. The quantum gravity encountering the tube would cause specific vibrations of the strings in the vessel. The increased vibrations would make the tube more massive, i.e. increasing gravitational influence by bending surrounding spacetime. The expanded hypothesis was based on the theory that increased string vibrations would be equivalent to increasing the energy of a body (Scherk, J. \& Schwarz, J. H., Nucl. Phys. 1974), resulting in an increased mass (Einstein, A., 1905). 
Therefore a graviton can be imagined as a mass-energy carrier, and the definition of mass is the vibration of strings. The graviton vibrations would have similarly influenced spacetime if considering that the manifold is a string field or brane. Again new theory is not being proposed, including the notion that dimensionality would have been crucial for the graviton to have been influential. In other words, because the gravitons would be produced in the same flat spacetime or critical dimension, the string bodies would be stronger, more influential than the strength typically presented for gravity, and could affect the tube or spacetime.

The laser studies for this paper had the light directly on the side of the graphite tube. Video captured spacetime bending and a multi-dimensional object that resembled a membrane with an open string at the position of where the light was traveling toward the tube. The studies confirmed a direct relation between fundamental frequency and string tension since only specific frequencies were effective in consideration of the magnetic fields.

Fig. 1 shows the experimental set-up; the instrument settings were the same for the preliminary laser work with the light near the tube. A camera (Kodak Easy Share C180) was clamped to a stand above the light on the tube at about 90-100 degrees and near $.01-.02 \mathrm{~m}$ in front of the outer edge of the tube toward the West side in relation to the magnet poles. The camera was near $.29-.3 \mathrm{~m}$ from the base of the stand to allow it to be as close as possible without touching the set-up or being in the path of the laser. The camera location should be altered if needing to improve recordings; zooming can be used to avoid adjusting the camera position on the stand.

The laser is not a component for the technique. The light was incorporated only to examine influences exiting the graphite tube. Thus, the method may be considered similar to NMR. The experimental set-up, particularly considering that the extremely low frequencies were not affecting Hydrogen alignments, should explain how the technique differs. The components that should be maintained consistently are the frequency and amplitude $(\approx 2 \mathrm{~Hz}$ $(2.000-2.012 \mathrm{~Hz}), \mathrm{V}_{\mathrm{p}-\mathrm{p}}=4.312-4.437$, predominately 4.375$)$, graphite tube, distance from the $\mathrm{S}$ pole to ensure a consistent 2000Gs, and the concentrated sulfuric acid (96\% concd, Mallinckrodt Analytical Reagent, ACS). Impurities should be limited in the acid supply, which should be replaced periodically. If replication at $2 \mathrm{~Hz}$ is unsuccessful, the amplitude should be examined. The setting would be less or more relative to a set-up change, e.g. different Hydrogen source or holding vessel.

\section{Results}

The images presented are the light from the laser in air that was a steady temperature and composition--i.e. consistent relative humidity, acid fumes, etc--for trials and control experiments to prevent factors as dust or refraction to affect the light. The work was conducted in a dark room; VLC media player was the imaging software used to capture frames from videos. What was captured on video suggested that light is related to open strings.

The first frame in Fig. 2 is the set-up. The subsequent frames were initially thought to be the laser light deflecting and remaining as a line along possibly the edge of a field. The light was later understood to have been the rotation and deforming of the D-brane. The events began at approximately nine minutes and eight to nine seconds and continued until nine minutes and fourteen to fifteen seconds. Appearances near the set-up may have been missed; the ideal method to see all proceedings involving the brane is to reduce the video speed. The lowest speed provided by the software $0.03 \mathrm{x}$ permitted the best views for capturing the images of Figs. 2 and 3 . Altering the camera position in future trials may permit additional observations.

The D-brane in the center frame emerged momentarily at nine minutes and nine or ten seconds. The curvature of the sides, seemingly tubular, meant that the rectangular image was multi-dimensional, apart from the singular wave-like string that was near the top. Research showed that influences from the tube caused two movements: a push followed by an attraction (pull), due to the bending of spacetime, to the tube. What was suspected to have caused the membrane to appear was the pushing influence from the tube, suggested in preliminary trials to involve the graviphoton, that stopped the light in position prior to it being rotated and stretched along the edge of a subsequent field that emerged from the graphite tube. The push was on spacetime, which resulted in the light being influenced.

Fig. 3 shows two images of the D-brane with the serpentine-like open string. The frames were not altered and are presented directly from video, apart from reducing the sizes to fit in this paper. The open string is in a slightly different position in Fig. 3b. Still, the end of the string seemed always in-line with the original position of the laser light on the tube.

Again the air was consistent to prevent refraction. The multi-dimensional image showed multiple structures similar to theorists' predictions. The image appeared momentarily prior to deforming for a couple of seconds before the light returned precisely to the initial position on the tube. Numerous control experiments including 
trials with schlieren photography never showed light to behave similarly, having multiple parts at different angles that seemed to be moving and have different dimensionality. Nothing passed in front of the laser; no alterations of the set-up occurred during trials. And videos of the light on the tube with the frequency off or simply the light passing in the magnetic field did not present the images of Fig. 3.

The influence of gravitons on the graphite tube subsequently bending spacetime can be seen with the curving of the light around the tube in Fig. 3. The curving is most appreciated with the contrasting Fig. 4. The slight light near the upper-right corner of Fig. $4 \mathrm{~b}$ was the reflection of the laser off the photocell surface. The reflection was common in frames and had no affect on the images of Fig. 3 or the deforming of the brane in Fig. 5.

The images for Fig. 4 were reduced to be the same size as the frames in Fig. 3, 4c was widened slightly, but no other alterations were made. Previously presented heights from the base of the stand are for the camera, not the related lens that extended below it. Different distances should be tried to improve visualizations, particularly since the heights may be useless depending on the experimenter's set-up: positioning of stand due to size and base shape, type and size of magnet, variety of camera, etc. Fig. $4 \mathrm{c}$ shows an adequate location of the clamp for the camera, though about $.005-.01 \mathrm{~m}$ lower than the position for $4 \mathrm{a}$, as earlier described to be in front of the set-up and as close as possible without touching the alligator clips that were later attached to the tube.

The preliminary trials had resulted in the triggering of the oscilloscope (Coupling=DC, Edge mode (negative edge), Trigger level $900 \mathrm{mV}$, Triggered Sweep) due to influences from the tube affecting spacetime and consequently the path of the light. However the triggering did not occur in the D-brane trial. The trigger negative edge should be adjusted to account for continued light entering the photocell $\left(\mathrm{R}_{\mathrm{L}}=100 \mathrm{k} \Omega\right)$ if triggering is required for future studies.

Fig. 5a shows light curving around the tube and the deformed D-brane to have maintained a distance from the tube. The observations suggested that a field had emerged to cause the brane in spacetime to maintain the close to parallel location, apart from simply due to the fixed position of the laser. The frame was reduced in size but was not altered otherwise. The frame shows brighter appearance of the light. The greater brightness compared to the original red light on the tube was likely evidence of redshifting, which occurred most after the initial appearance of the D-brane and was thought to have coincided with the interacting of gravitons with the tube and the emergence of the suspected graviton field. The line of light ended with it returning toward the original position of the D-brane before reappearing at the same point on the tube when the trial began. To see properly each occurrence, again the video should be slowed as much as possible.

Light seemed to be trapped having entered the tube. Observations suggested that graviton influence caused a portion of the acid medium to become an increased gravitational point similar to a black hole, which gradually disappeared. The light continued to be on the tube, curved but inconsistent around it when the increased gravitational point existed. The light curved to the increased gravitational region that was at a specific location in the tube. While the laser remained on, the open string continuously provided the light on the vessel. Because of the camera positioning, all sides of the tube were unclear. Thus, light in some amount may have been at an unobservable part. Fig. 4b was compared to the curving in various frames and more light never seemed to be on the lower right side of the light. If light had been completely circling the tube, additional laser light likely would have been noticed at the beginning of the lower right side. The gravitational region was possibly absorbing the light.

The patch of trapped light was greatest near the time of the emergence of the brane. The pushing had already occurred while the gravitons began to be influential. The coinciding of the amount of light and the appearance of the D-brane supported previous observations of the pushing being followed by the graviton influence. D was not labeled in the upper right frame of Fig. $5 \mathrm{~b}$ to show the patch later with less light. Though light was captured by the possible black hole the gravitons were not trapped and traveled away from the point of emergence, resulting in the field or simply having influence away from the tube.

The rotation of the black hole seemed to increase after the D-brane had deformed, moving the brane further away from its original position seemingly faster at about nine minutes and ten or eleven seconds, the time being eight minutes and fifty-nine seconds in the video. The observation suggested that the black hole may have gained energy or angular momentum from the light and possibly from particles it may have been releasing (Hawking, S.W. 1975). Increased rotation due to the laser light would confirm that the light had entered the tube.

The deformed D-brane seemed to move to approximately $.013-.014 \mathrm{~m}$ before returning to the previous line position of about $.01 \mathrm{~m}$ from the tube. The movement suggested multiple productions of the pushing influence, including a gravitational wave. Gravitons and the associated field maintaining the position of the line of light on the West/Northwest side (in relation to the magnetic field) indicated that the original thought of influence being 
only or primarily from the S pole side of the tube was not entirely correct. The influences of the gravitons were from the tube generally, the field consequently being in multiple if not all directions. The $\mathrm{S}$ pole conclusion had occurred due to preliminary trials including Ge detector work that suggested radiation resulting from the technique to produce gravitons was emerging from the side at the $\mathrm{S}$ pole.

A pushing initially emerged from the tube to influence spacetime thereby affecting the light. A subsequent field remained for seconds, gradually dissipating. Because the position of the deformed brane seemed straight, the field was thought filled or formed a brane, the light being along a side of the dimensional space with width. Filling was suspected, possibly the brane having emerged from the initial push.

The appearance of the D-brane was unexpected, especially that it appeared before ten minutes. Though graviton formation could occur earlier than nine minutes, any possible influences on the light were thought would happen at approximately thirty minutes or near forty to fifty minutes when the greatest effects had been observed. The trial showed that significant affects from the tube could occur at any time, in keeping with the understanding that influences emerged gradually and could be periodic.

By increasing the brightness of the video somewhat and setting the speed to $0.25-0.5 x$, the images of Fig. 5 appeared primarily to have been the side of the D-brane closest to the observer, D in Fig. 5b. The D-brane rotated slightly and deformed with D stretching. The bright, upper endpoint of the line in Fig. 5a was the corner of the brane closest to the observer in Fig. 3, A in Fig. 5b.

Frames were omitted for Fig. 5b, intended simply to explain the brane deformation. The cause of the rotation seemingly was spacetime being rotated or dragged around the previously mentioned gravitational point, i.e. frame-dragging. The D-brane was fixed in place due to the consistency of the laser and was in spacetime, separate than the field from the tube that also moved: in the direction of the rotation of the increased gravitational area. The end of the serpentine-like open string B could still be seen as the brane deformed. And C was the top edge of the D-brane.

An attempt to trace $\mathrm{C}$ is in the last frame of $5 \mathrm{~b}$. Considering $\mathrm{C}$ was still connected to $\mathrm{A}, \mathrm{C}$ disappearing behind $\mathrm{D}$ suggested that D had stretched, which further can be appreciated by noticing the changing position of $\mathrm{A}$ especially in reference to the curved light to the right: the tube that was held in place. The D-brane in the last frame seemed to be closer to the tube. Again the light, labeled Photocell, on the opposite side in the frames was from the photocell surface.

The light in the increased gravitational area when the D-brane was present appeared to have a mist-like appearance, similar to the description of a fuzzball (Lunin, O. \& Mathur, S.D. 2002). The method of this paper that led to a symmetry breaking for graviton emergence also could have allowed for the strings necessary for a unique fuzzball, due to what comprised the production involving electron capture in Hydrogen. The increased gravitational point was the complete region that has been described as the trapped light. Brightened more than Fig. 5b, Fig. 5c is a frame showing that side D had moved unevenly closer to the increased gravitational point, which is designated with the white oval. D was traced to show that the middle was nearer; the brightening had permitted the slight bending to be noticed.

The patch of light appeared more on the inside of the tube, as though the tube wall had not been a barrier. The light was near $.0127 \mathrm{~m}$ in the tube from the inner edge. The increased gravitational point may have existed at a higher location in the tube, closer to the top of the $20 \mathrm{~mL}$. Tracing the increased gravitational region and bent line was difficult but was primarily to provide an idea of the oval shape in the tube while the curved light disappeared in it, the increased gravitational point suspected to have caused the light to pass through the side of the vessel. The difficulty in tracing the oval region is the only reason for the different sizes of the Fig. $3 \mathrm{a}$ and $5 \mathrm{c}$ ovals.

Redshifting, greatest near the increased gravitational point, would explain the light entering the tube. The increased wavelength due to the gravitational influence would have permitted the light to fall to the area by passing through the tube wall, which exposed the greater strength of quantum gravity than what has been accepted for gravity. The continued observation of the light as in the initial frame of the D-brane did not occur since it was absorbed by the area. After the initial D-brane appearance, the graviton field extended beyond the tube.

In the D-brane trial, the light curvature disappearing meant that the imparted graviton influence had not been maintained, which suggested that a source existed for the quantum gravity, likely the increased gravitational point. As it dissipated so did the continued exchange or supply of the gravitons. The observation supported that the point was a black hole. Still, the source of gravitons in a trial needed not be a black hole. Gravitons could simply emerge from the Hydrogen procedure that would also result in neutron productions, observed in 
preliminary trials. Specifically, the technique of this paper led to electron capture and resulting isotope production by neutron capture. The neutrons and gravitons seemed to occur near or exactly at the same times, which suggested that a symmetry breaking was involved for both productions.

Apart from camera position, the most crucial factors for observations, understood only after repeated trials, were low barometric pressure and high relative humidity, about $99.7 \mathrm{kPa}$ (Boston, MA, CW1378) and $98-100 \%$ on the day of the Fig. 5 brane. Air pressure and density could create a barrier against the tube that would restrict the influences from emerging and affecting spacetime. Accordingly, greater light movements were seen on days having both low barometric pressure and high relative humidity. An available vacuum chamber was tried but was too small for the magnet or did not allow for proper camera positioning even when incorporating a smaller $1 \mathrm{kGs}$ magnet, which led to reliance on weather forecasts. Light movements could be observed with the relative humidity being low if the barometric pressure was also low. No observations were recorded on days with relative humidity being lower than $65-67 \%$, even with low barometric pressure. The ideal situation for the barometric pressure was to have it stable over a few hours to an entire day, though fluctuating pressure--decreasing quickly and increasing rapidly shortly afterward--could permit visualizations. The pressure needed not be below $100.0 \mathrm{kPa}$ for observations, though the D-brane was unobserved above $99.7 \mathrm{kPa}$.

\subsection{Intensifying the influences from the tube}

Observations were not detected at higher barometric pressures unless exposing the acid to direct current (DC) to free more Hydrogens prior to using the $2 \mathrm{~Hz}$. By that, the source of a graviton was a Hydrogen; results from preliminary studies implied that greater influences from gravitons and related bodies could be produced by increasing the number of free Hydrogens in the magnetic field prior to $2 \mathrm{~Hz}$ exposure.

On a day atmospheric pressure was $100.4 \mathrm{kPa}$, the acid was exposed to DC (set to $2.9 \mathrm{~V}$ and $5.150 \mathrm{~A}$, readings during trial were $0.623 \mathrm{~V}$ and $5.092 \mathrm{~A}$ ) by attaching alligator clips to the graphite tube for an hour prior to replacing it with the $2 \mathrm{~Hz}$ for the typical hour as for trials without DC. A brighter amount of light seemed to occur on the tube to the right of the original laser light placement. Light movements were not recorded with the same camera position when the acid was exposed to DC on days when the pressure was $100.7-100.8 \mathrm{kPa}$ or higher. Again light movements may have occurred but conclusions could only be made if events were captured on camera. Observations from the $100.4 \mathrm{kPa}$ trial reiterated that two separate influences appeared; an affect split the light before an influence caused the light to be highly concentrated at the emergence of the previous affect. The same body may have been responsible for the influences; yet, again preliminary work suggested that different particles were surfacing.

The resulting greater influences from the vessel permitted the light observations by overcoming the relatively high barometric pressure and low relative humidity (near 65\%). A different brand of sulfuric acid (Duda Diesel, $98 \%$ concd.) was incorporated for the trial, which confirmed that the acid simply should be concentrated and lack excessive impurities. Control trials with the same conditions but without the direct current did not result in similar observations. The camera was located approximately $.3-31 \mathrm{~m}$ from the base of the stand and $.054 \mathrm{~m}$ more toward the $\mathrm{N}$ pole and $.02 \mathrm{~m}$ more in front of the magnet than when the full $\mathrm{D}$-brane was recorded. The location was simply to record from a new position and was unlikely the reason the D-brane was not observed. The recording of a brane still occurred with a different camera location than for the brane trial of this manuscript, the presented frames having been the clearest captures of a brane from a trial. The Fig. 3 D-brane was the largest brane recorded; a greater pushing influence due to more graviphotons having emerged, associated with a larger number of produced gravitons in the trial, was thought the reason.

In the DC trial the pushing influence, affecting spacetime at the point of emergence, can be thought to have been a barrier that divided the light, which recombined and redshifted due to subsequent graviton emergence at the splitting. Spacetime could not be influenced significantly due to the air pressure and density for the D-brane to be observed. The light being atypically initially dimmer on the tube due to weaker batteries in the laser factored in the observing of the increased intensity. If the initial light had been optimally bright, a change of intensity may not have been observed. The observations were due to influences from the tube and not the result of anomalous behavior by the laser, confirmed with specific control trials.

Increased gravitational influence therefore could happen at any point in the tube. The light was near the S pole, slightly toward the $\mathrm{W}$ side; thus, events from the tube may have interacted with the $\mathrm{S}$ pole of the magnet. Considering from previous observations that the area of the black hole could have been proportional to the larger region of light on the tube, the increasing size of the region for the trial suggested that a possible black hole was stretching, i.e. the diameter of the light grew as the black hole expanded. The increased light with growing diameter lasted four to five seconds, which could have been further evidence of extra spatial dimensions since the evaporation of a black hole may have been slowed (Keeton, C.R. \& Petters, A.O. 2006). 


\section{Conclusions}

Between nine minutes sixteen seconds and nine minutes eighteen seconds, approximately two to four seconds after the laser light returned to the original position on the tube in the D-brane trial, a sound similar to--depending on interpretation--static, a heartbeat, thumping, or tapping was recorded with the microphone of the camera. Due to the timing, the sound was suspected to have been the pressure wave created after the spacetime around the tube returned to its original state before light movements. The position of the camera was crucial for the recording. The noise was not recorded in various control trials. An alternative source was not found; attempts at mimicking the sound were unsuccessful.

Viewing the D-brane can be difficult. To improve the possibility of an observation the light should be grazing the tube, not on the vessel excessively. For replication purposes and to ensure consistency, the photocell can be used to align the light on the tube, even if the oscilloscope might not be triggered.

A difference must be understood between the laboratory work of this manuscript and events in nature. Fig. 1 is an arrangement for low energy trials that permits the study of strings. Data collected in the laboratory were unique to the method and should not be thought equivalent to occurrences with the same names in nature. For example, the mentioned black holes were due to gravitons that emerged from the Fig. 1 technique and not to the collapse of a star as outside of the lab.

The events related to graviton emergence including the possible black holes and the D-brane were suggestive of extra dimensions. Since preliminary work proposed that the pushes involved massive, limited range graviphotons, observations may have been evidence for superpartners. Accordingly related to supergravity, gravitino production was likely. Influences from graviphotons would suggest that a 2-brane can be a solution for quantum M-theory ( $\mathrm{D}=11$ ) (Billó, M. et al. 2000, Duff, M.J. \& Stelle, K.S. 1991, Bergshoeff, E., Sezgin, E., Townsend, P.K. 1987) that compactifies ( $\mathrm{D}=10)$ (Huq, M. \& Namazie, M.A. 1985, Hořava, P. \& Witten, E. 460B 1996) with eleven dimensional supergravity being the minimal energy limit (Hořava, P. \& Witten, E. 475B 1996).

Push and pull have been descriptions of observations caused by products of the technique; the words should not be considered an attempt to displace any theory as General Relativity, which this work supports and expands to quantum gravity. This paper affirms various unifications, including the unification of the forces of nature. The timing of the brane shown above was unexpectedly early, but other visualizations of the light coincided with the typical times of possible graviton productions in preliminary trials. For instance, the increased light on the tube on the $100.4 \mathrm{kPa}$ day appeared between fifteen and sixteen minutes and light movements in other trials were near fifty minutes, which again was when typically the greatest influences had occurred.

Continuous effort with low barometric pressure, high relative humidity, and the maintenance of procedure should allow for visualizations. The graphite tubes were the same vessels for previous isotope productions, the tube in the D-brane trial having been used to produce elements from Tungsten powder. Unintentional isotope production due to remaining Tungsten in the tube or simply involving Hydrogen from the acid was unlikely to have contributed directly to observations.

The novel method of this paper marks a paradigm shift since it allows for facile laboratory studies previously thought possible only with particle accelerators and enormously high initial energies. Issues were intentionally not greater discussed to avoid distraction from the main purposes of describing the technique and how to record a D-brane. For example, the possible black holes could be discussed as having been productions of dark matter (Keeton, C.R. \& Petters, A. O. 2006) in the laboratory. However, the disappearances of the black holes make the consideration illogical unless discourse would involve the possibility of dark matter evaporation. The technique can be utilized to study aspects of this work in more detail or to understand topics similar to events in this paper. For instance, motivated by the pushing influence at different times during the D-brane trial, the method can be used to investigate possible gravitational waves from the experimental set-up or to discuss topics as dark energy or negative pressure and the dilaton in addition to the graviphoton, i.e. multiple causes for pushes. Different black holes in the acid permitted unique sources of gravitational waves.

\subsection{Supplementary files}

See the supplementary files for the videos of the brane (braneFIG58) and the light movement after DC was applied (DCsectionIIIA15). The first eight minutes were removed from braneFIG58 to reduce the video size. The above-mentioned sound that occurred after the brane can still be heard. The times mentioned for the brane video are the readings from a stopwatch that was started as soon as the frequency for the technique was turned on, which slightly preceded the operation of the camera and explains the time differences between the appearances on the video and what is reported. Fifteen minutes should be added to DCsectionIIIA15 when viewing it because the minutes were deleted to reduce file size. If using VLC media player, the Magnification/Zoom tool can be helpful for examinations. 


\section{References}

Bergshoeff, E., Sezgin, E., Townsend, P.K. (1987). Supermembranes and eleven-dimensional supergravity. Phys. Lett., 189B, 75-78, http://dx.doi.org/10.1016/0370-2693(87)91272-X

Billó, M. et al. (2000). Shadow multiplets in $\mathrm{AdS}_{4} / \mathrm{CFT}_{3}$ and the super-Higgs mechanism. Nucl. Phys., 591B, 139-194, http://dx.doi.org/10.1016/S0550-3213(00)00532-0

Duff, M.J. \& Stelle, K.S. (1991). Multi-membrane solutions of $D=11$ supergravity. Phys. Lett., 253B, 113-118, http://dx.doi.org/10.1016/0370-2693(91)91371-2

Einstein, A. (1905). Ist die Trägheit eines Körpers von seinem Energieinhalt abhängig? Annalen der Physik. 18, 639-643, http://dx.doi.org/10.1002\%2Fandp.19053231314

Hawking, S.W. (1975). Particle creation by black holes. Commun. Math. Phys., 43, 199-220, http://dx.doi.org/10.1007/BF02345020

Hořava, P. \& Witten, E. (1996). Heterotic and Type I string dynamics from eleven dimensions. Nucl. Phys., 460B, 506-524, http://dx.doi.org/10.1016/0550-3213(95)00621-4

Hořava, P. \& Witten, E. (1996). Eleven-dimensional supergravity on a manifold with boundary. Nucl. Phys., 475B, 94-114, http://dx.doi.org/10.1016/0550-3213(96)00308-2

Huq, M. \& Namazie, M.A. (1985). Kaluza-Klein supergravity in ten dimensions. Class. Quant. Grav., 2, 293-308, http://dx.doi.org/10.1088/0264-9381/2/3/007

Keeton, C.R. \& Petters, A.O. (2006). Formalism for testing theories of gravity using lensing by compact objects. III. Braneworld gravity. Phys. Rev., 73D, 104032, http://link.aps.org/doi/10.1103/PhysRevD.73.104032

Lunin, O. \& Mathur, S.D. (2002). AdS/CFT duality and the black hole information paradox. Nucl. Phys., 623B, 342-394, http://dx.doi.org/10.1016/S0550-3213(01)00620-4

Scherk, J. \& Schwarz, J.H. (1974). Dual models for non-hadrons. Nucl. Phys., 81B, 118-144, http://dx.doi.org/10.1016/0550-3213(74)90010-8

Scherk, J. \& Schwarz, J.H. (1974). Dual models and the geometry of space-time. Phys. Letters., 52B, 347-350, http://dx.doi.org/10.1016/0370-2693(74)90059-8

Thorlacius, L. (1998). Introduction to D-Branes. Nucl. Phys. Proc. Suppl., 61A, 86-98, http://dx.doi.org/10.1016/S0920-5632(97)00521-5

Zwiebach, B. (2004). A first course in string theory. Cambridge: Cambridge University Press.

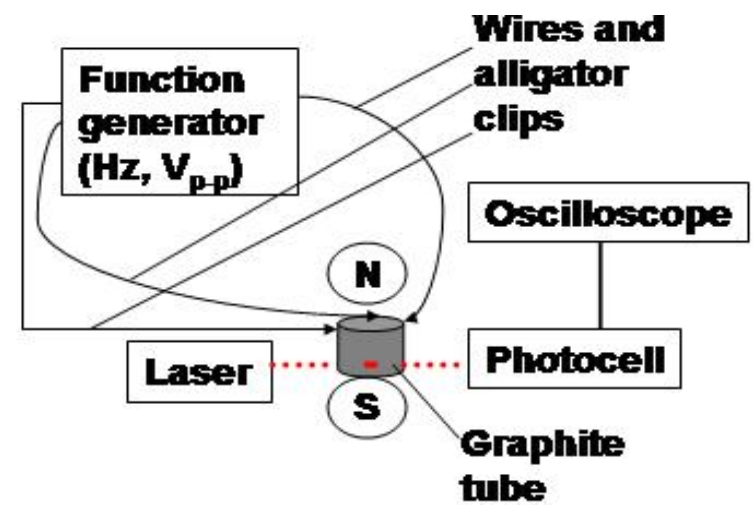

Figure 1. Experimental set-up

The light from the laser (Quartet Standard Laser Pointer) was positioned on the S pole side of the tube, approximately $.017 \mathrm{~m}$ from the center and $.01-.02 \mathrm{~m}$ from the bottom of the tube. The positioning created an $\approx .004-.005 \mathrm{~m}$ line on the tube but did not prevent the light from entering a vacuum photocell that was connected to a digital oscilloscope as diagrammed. The same number of wooden blocks was placed under the stand for the laser and the photocell to raise the objects for alignment. The tube should be clamped in the area of the magnetic field measuring approximately $2000 \mathrm{Gs}$. Slight distance differences toward the S pole were not a factor. Past trials had the tube $\approx 0.01-0.012 \mathrm{~m}$ from the $\mathrm{S}$ pole. For the trial that presented the D-brane, the distance was $.004-.005 \mathrm{~m}$; 
the tube was angled near five degrees toward the $\mathrm{S}$ pole. The laser was $.34-.35 \mathrm{~m}$ from the tube. The function generator was connected to a LED on a breadboard from which wires provided a specific frequency and amplitude to the graphite tube. Three alligator clips were attached to the top of the tube in a triangular formation, though only one wire was needed to provide the $4.375 \mathrm{~V}$ amplitude. The three wires were maintained to ensure that the frequency and amplitude were reaching the tube, particularly considering possible malfunction during a trial as due to corrosion of a clip. Frequencies and amplitudes were confirmed with an oscilloscope prior to each trial. The laser was kept on by a clamp during all experiments, and the batteries were changed before every trial to guarantee steadiness of the light, unless not changing the batteries was procedural. The photocell was $.63-.64 \mathrm{~m}$ from the graphite tube, which was approximately $.098-.099 \mathrm{~kg}$. The clamp that held the tube was about $.230 \mathrm{~kg}$, and the stand to which the clamp was attached was near $1.458 \mathrm{~kg}$, which included the mass of the clamp holder that was $\approx .153 \mathrm{~kg}$.

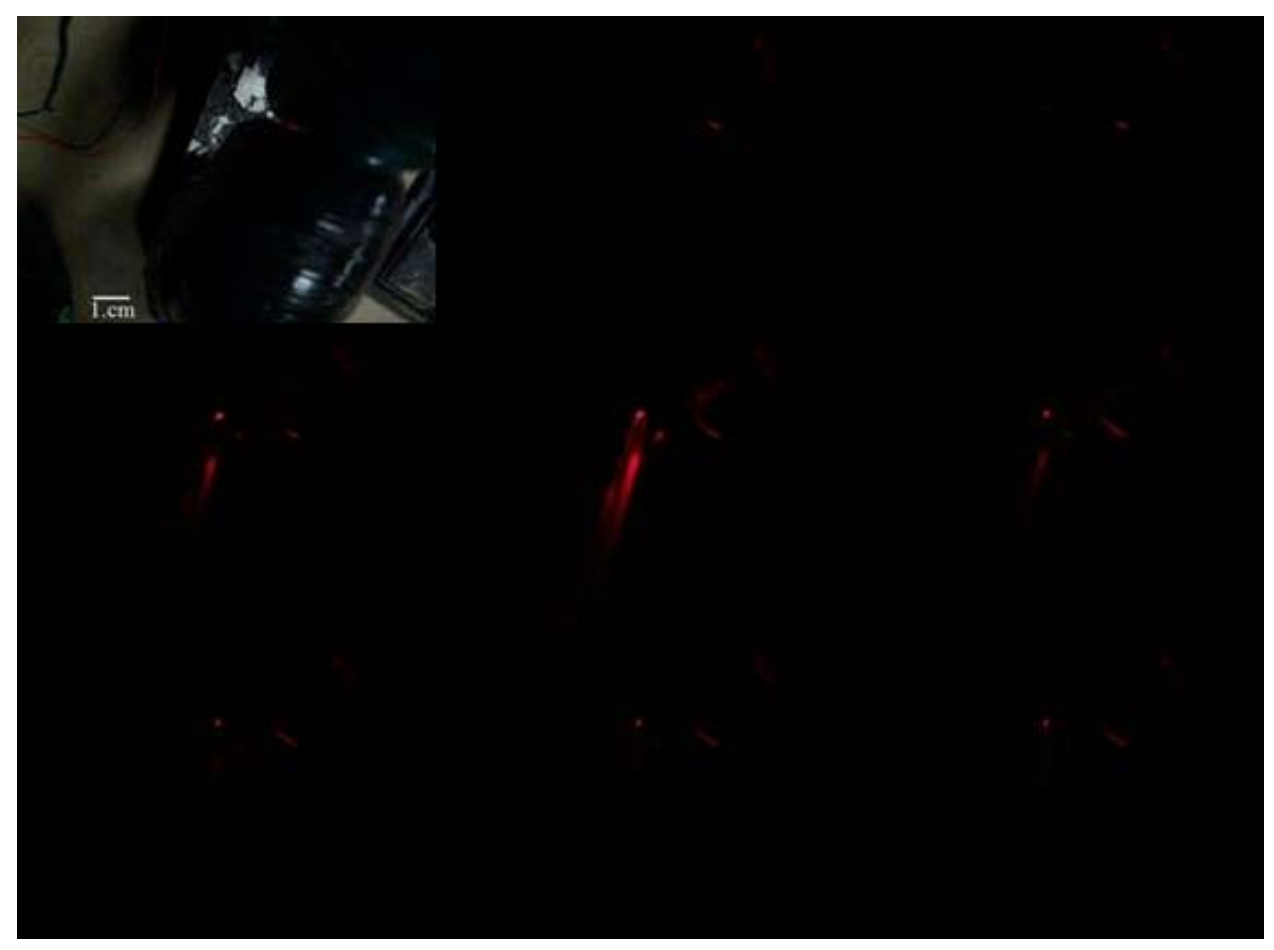

Figure 2. Sequence from video leading to the D-brane and shortly afterward

The frames from left to right show the progression to the D-brane, the center frame that is better presented in Fig. 3 , and the beginning of the deforming of the brane, the subsequent frames. No alterations, e.g. rotation or color, were made. The frames were simply reduced in size to fit in Fig. 2. 
a

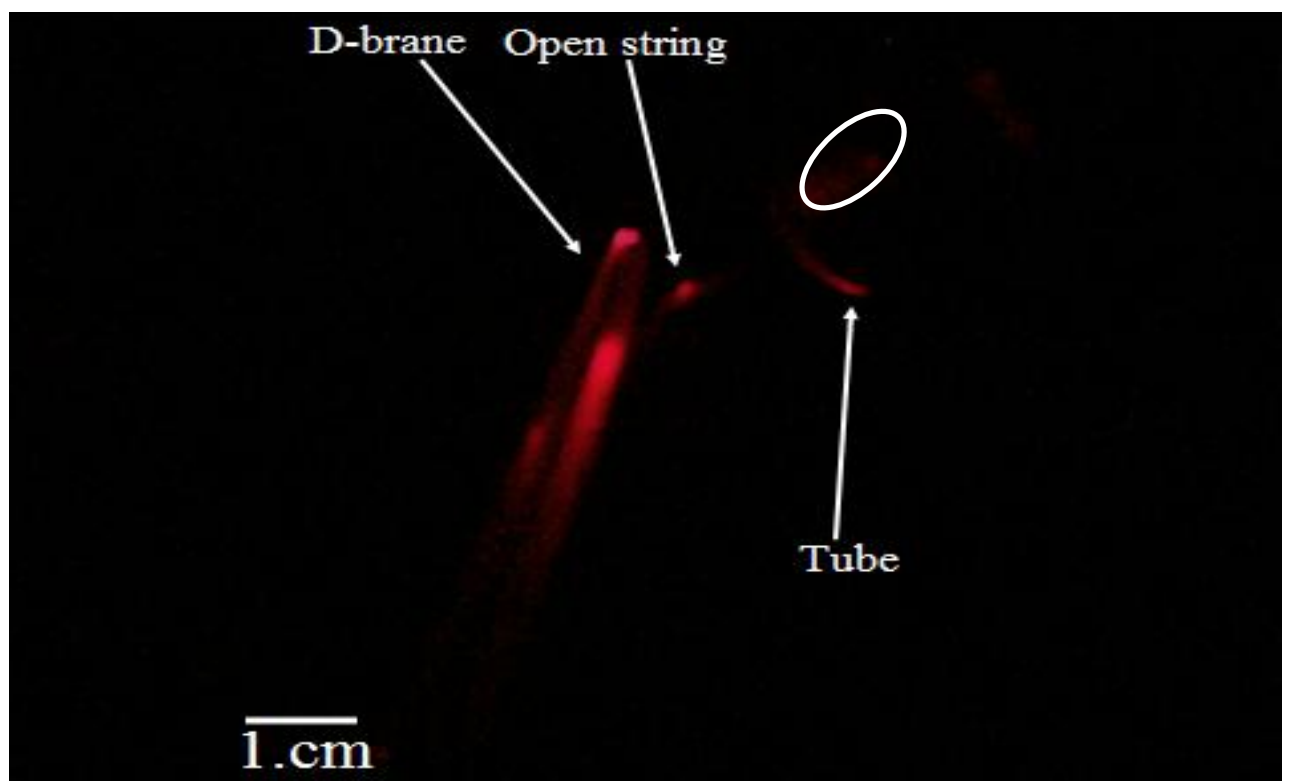

b

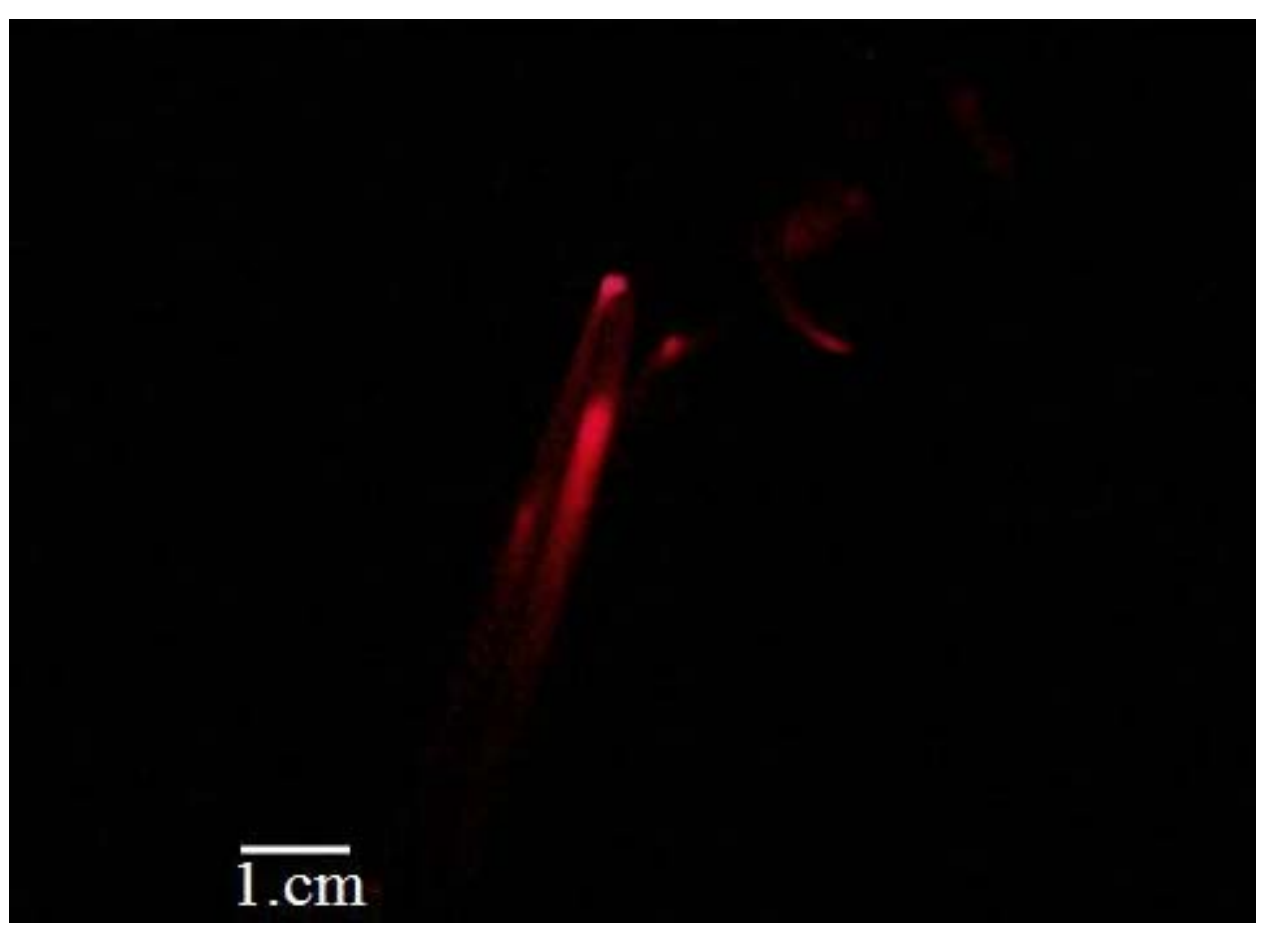

Figure 3. D-brane progression

a, Initial full appearance of the D-brane with the end of the open string in-line with the position of the laser on the tube. b, Proceeding frame with the open string in a slightly different position. 
a

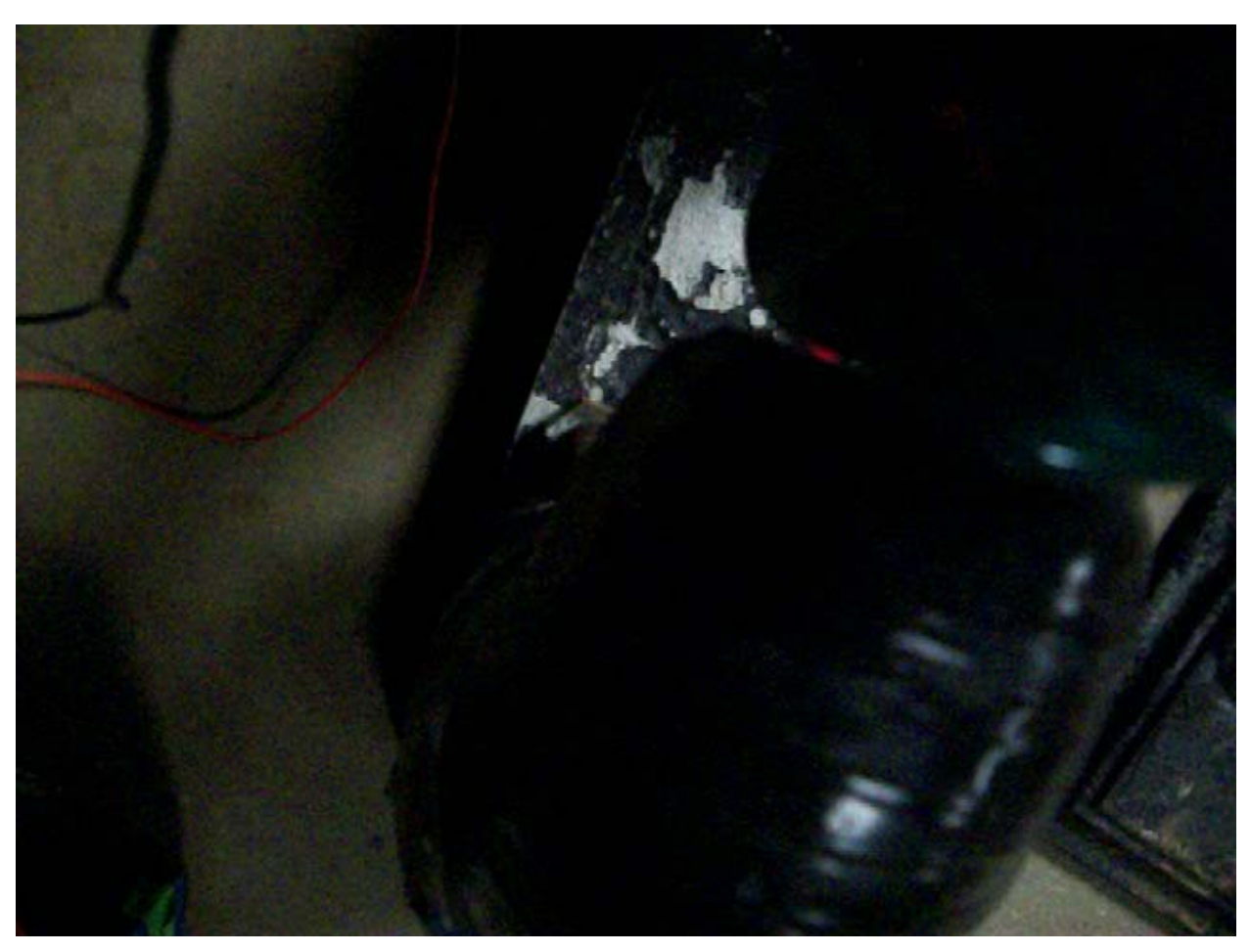

b

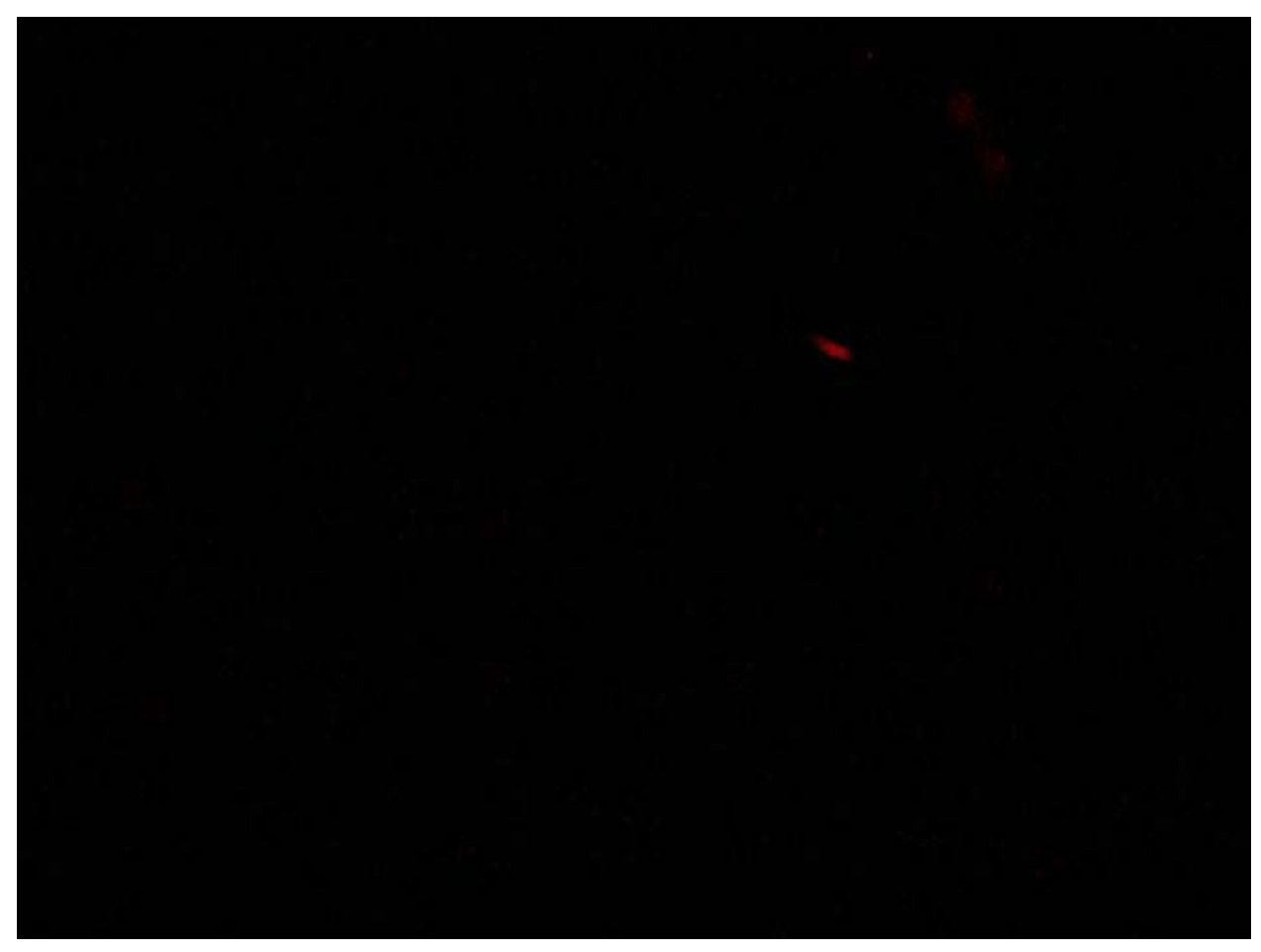


C

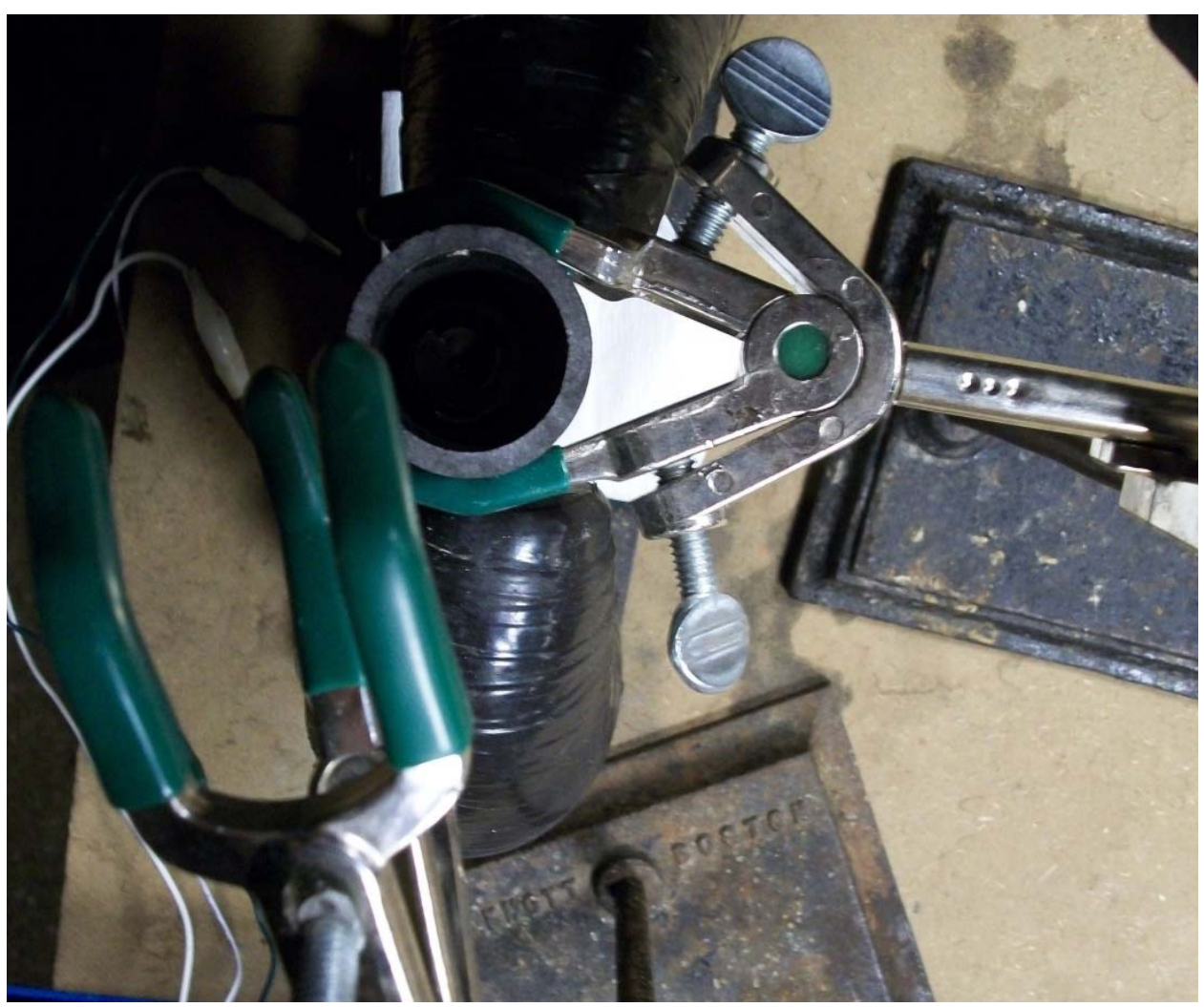

Figure 4. Light before influences from the tube in the brane trial

a, The light is seen on the tube while the ceiling lights are on. $\mathbf{b}$, With the ceiling lights off, the laser light is on the tube prior to influences. c, The stand that held the camera above the tube is shown in an approximate position for trials. 
a

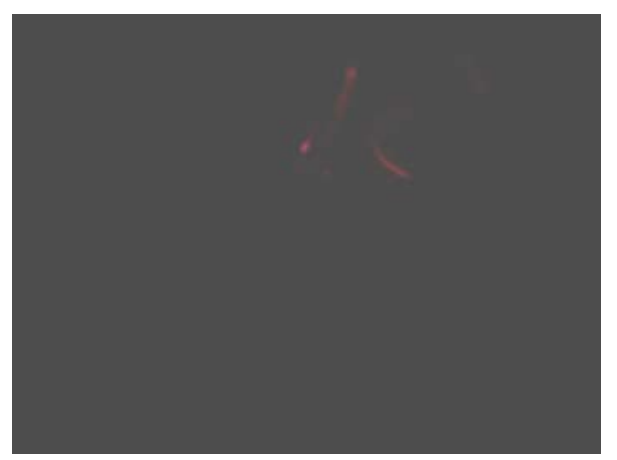

b

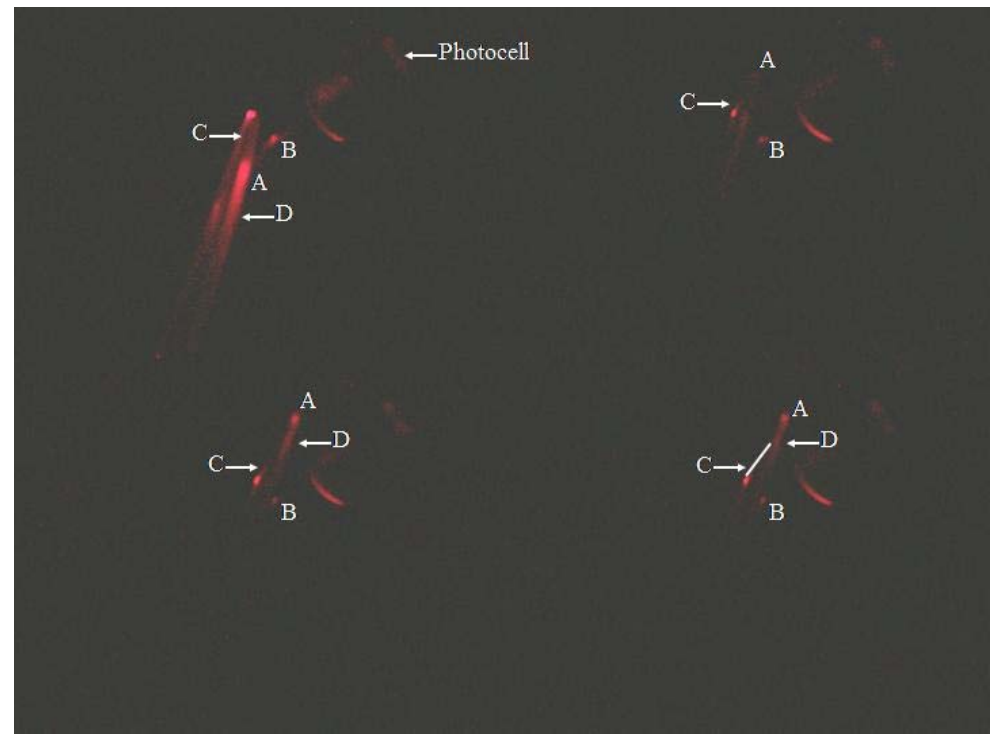

C

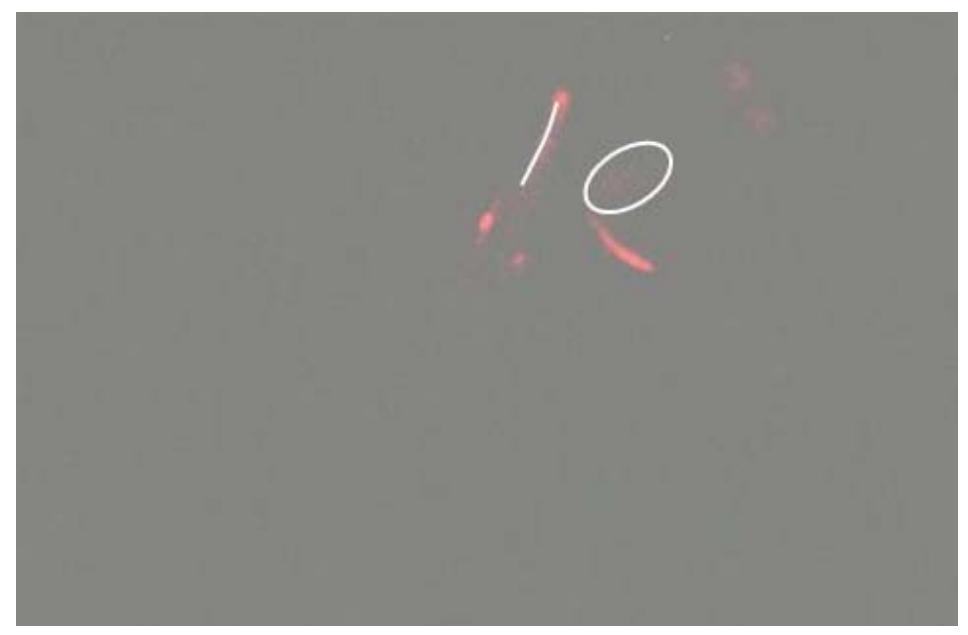

Figure 5. Deforming of the D-brane

a, A more intense image of the deformed D-brane is shown as it appeared as a line nearly parallel to the magnet and tube set-up. b, The sequence from left to right is the D-brane along the edge of a possible field. The D-brane is presented to be deforming in relation to the tube until the brane becomes the shape of 5a. c, A brighter frame of the deformed D-brane is presented. An increased gravitational area or point is marked with an oval while side $\mathrm{D}$ of the D-brane is traced showing that it is attracted unevenly to the oval. 